\title{
Detección de papilomavirus humano en mucosa oral en hombres con verrugas anogenitales
}

\author{
Marcelo Gabriel Medina ${ }^{1}$ (D) Karina Marinic $^{2}$ (D) Myriam Lucrecia Medina $^{3}$ (i) , Alicia \\ Sorrentino $^{2}$ iD, Manuel Fernando Giménez ${ }^{4}$ iD, Luis Antonio Merino ${ }^{1}$ (D) \\ ${ }^{1}$ Universidad Nacional del Nordeste, Instituto de Medicina Regional. Av. Las Heras 727. \\ Resistencia, Argentina \\ ${ }^{2}$ Hospital "Dr. Julio C. Perrando". Av. 9 de Julio 1100. Resistencia, Argentina \\ ${ }^{3}$ Hospital “Dr. Avelino L. Castelán”. Av. Vélez Sarsfield 120. Resistencia, Argentina \\ ${ }^{4}$ Centro Dermatológico “Dr. Manuel M. Giménez". Julio E. Acosta 300. Resistencia, Argentina
}

Cómo referenciar este artículo/ How to reference this article:
Medina MG, Marinic K, Medina ML, Sorrentino A, Giménez MF, Merino LA. Detección de papilomavirus humano en mucosa oral en hombres con verrugas anogenitales. Mem. Inst. Investig. Cienc. Salud. 2021; 19(2): 41-48

\begin{abstract}
RE S U ME N
El virus del papiloma humano (HPV) es el agente etiológico de infecciones de transmisión sexual relacionadas con procesos oncogénicos genitales y orales. La biología molecular, mediante la identificación de los tipos virales involucrados, proporciona precisión diagnóstica con un enfoque epidemiológico. El objetivo de este estudio fue determinar la presencia de HPV en la mucosa oral de hombres con verrugas anogenitales y correlacionar los genotipos detectados en ambas muestras. Se estudiaron 26 pacientes varones con verrugas anogenitales que acudieron al Instituto de Dermatología de la ciudad de Resistencia (Argentina). La presencia de HPV en muestras orales y anogenitales se estudió mediante reacción en cadena de la polimerasa (PCR) y la genotipificación se realizó mediante PCR-RFLP. La prevalencia de HPV oral en pacientes con HPV anogenital fue del 46,2\% (12/26). Se encontraron dieciocho genotipos con alto riesgo oncogénico en muestras orales de pacientes con infecciones únicas o múltiples y el tipo 16 fue el más frecuente ( 6 pacientes). Catorce genotipos en muestras orales fueron de bajo riesgo oncogénico, el más frecuente fue el tipo 6 (10 pacientes). En muestras anogenitales el genotipo 6 fue el más frecuente (13 pacientes), solo o en coinfección. Se encontró una alta prevalencia de HPV oral de malignidad de alto grado en nuestra población y coinfección con tipos oncogénicos. Las prácticas de sexo oral fueron la principal conducta de riesgo para la infección, lo que quedó demostrado por el hallazgo simultáneo del mismo tipo de HPV en muestras orales y anogenitales.
\end{abstract}

Palabras clave: HPV, hombres, verrugas anogenitales, mucosa oral.

\section{Detection of human papillomavirus in oral mucosa in men with anogenital warts}

Fecha de recepción: Mayo 2021. Fecha de aceptación: Junio 2021

* Autor correspondiente: Luis A. Merino. Chaco 1163 6B. 3400 Corrientes. Argentina

Teléfono: +54 3794632760

Email: luisantoniomerino@gmail.com 
both samples. Twenty-six male patients suffering anogenital warts who attended to the Institute of Dermatology in Resistencia (Argentina) were studied. The presence of HPV in oral and anogenital samples was studied by PCR and genotyping was performed by PCR-RFLP. The prevalence of oral HPV in patients with anogenital HPV was $46.2 \%(12 / 26)$. Eighteen genotypes with high oncogenic risk were found in oral samples of patients with single or multiple infections and type 16 was the most frequent ( 6 patients). Fourteen genotypes in oral samples were of low oncogenic risk and the most frequent was type 6 (10 patients). In anogenital samples the genotype 6 was the most frequent (13 patients), alone or in co-infection. A high prevalence of high-grade malignancy oral HPV in our population and co-infection with oncogenic types were found. Oral sex practices were the main risk factor for infection, which was demonstrated by the simultaneous finding of the same HPV type in oral and anogenital samples.

Keywords: HPV, men, anogenital warts, oral mucosa.

\section{NTRODUCCIÓN}

El Virus del Papiloma Humano (HPV) es un virus con una doble hebra de ADN circular y en los seres humanos se han aislado y caracterizado molecularmente cerca de 100 tipos y de éstos, alrededor de 40 infectan el tracto anogenital y aerodigestivo superior $^{(1)}$. El HPV es el agente etiológico de la infección de transmisión sexual más frecuente en el mundo. El $70-80 \%$ de las mujeres y los hombres sexualmente activos han estado expuestos al virus en algún momento de su vida. Se estima la existencia de 310 millones de portadores de HPV, lo que tiene una gran importancia sobre todo si se considera la asociación que existe entre la infección por algunos tipos de HPV y la aparición de carcinomas anogenitales y de la cavidad orofaríngea ${ }^{(2)}$.

De acuerdo con el riesgo oncogénico, la clasificación de los HPV no está definida totalmente, pero según Muñoz y cols. son de bajo riesgo los tipos $6,11,40,42,43$, $44,54,61,70,72,81$ y de alto riesgo los tipos 16, 18, 31,33, 35, 39, 45, 51, 52, 56, $58,59,68,73$ y 82 . Los tipos 26, 53 y 66 deben considerarse probables carcinógenos $^{(3)}$. La infección por HPV de bajo riesgo, principalmente los tipos HPV 6 y 11, producen lesiones benignas anogenitales y papilomatosis laríngea o infecciones subclínicas que suelen ser transitorias y casi siempre se resuelven sin secuelas, mientras los de alto riesgo se asocian con displasias y carcinomas anogenitales y cervicales en el caso de infecciones persistentes ${ }^{(4)}$.

El cáncer bucal es una patología de etiología multifactorial pero hay que destacar que durante los últimos años se puso en evidencia la implicación del virus del papiloma humano en el desarrollo de lesiones precancerígenas y del carcinoma escamocelular de la cavidad bucal ${ }^{(5)}$. La presencia del virus del papiloma humano en la cavidad bucal ha sido clasificada en dos grandes grupos: lesiones benignas y lesiones premalignas o malignas. Entre las lesiones bucales benignas se incluyen el papiloma bucal, verruga vulgar bucal, condiloma acuminado bucal e hiperplasia epitelial focal (también llamada enfermedad de Heck); las lesiones premalignas o malignas incluyen la leucoplasia y el carcinoma escamocelular ${ }^{(6)}$. El ADN del HPV ha sido demostrado en algunos carcinomas de células escamosas ${ }^{(7)}$; sin embargo, hay poca información de la prevalencia en la mucosa bucal clínicamente sana ${ }^{(8)}$.

Existe un notable incremento de la infección por HPV en el mundo y escasez de estudios de este virus en la cavidad bucal y en población masculina, la cual es considerada reservorio de la infección por HPV por su directa influencia en la carcinogénesis cervical.

Los estudios sobre HPV en zonas con diferentes tasas de incidencia contribuyen al conocimiento de la historia natural de la infección, de sus enfermedades asociadas y de los factores de riesgo, aportando información necesaria para el desarrollo de vacunas efectivas y el diseño de programas de prevención del cáncer cervical, anogenital y bucal $^{(9)}$. Además, la detección temprana de las lesiones bucales precancerosas asociadas a virus del papiloma humano es muy importante para el tratamiento oportuno del paciente. 
En los últimos años se ha demostrado que la infección por HPV en el hombre puede causar diversas patologías en estos individuos, así como también en sus parejas sexuales. Por ello cobra relevancia la necesidad de la detección y genotipificación de VPH en población masculina, para instaurar programas de prevención de la transmisión del virus ${ }^{(10)}$.

El objetivo de este trabajo fue detectar la presencia de HPV en muestras de mucosa oral de hombres con verrugas genitales, genotipificarlos y correlacionar los genotipos hallados en ambas localizaciones.

\section{MATERI ALES Y MÉTODOS}

Pacientes: en el período de un año se estudiaron todos los hombres que consultaron por verrugas anogenitales al Centro Dermatológico “Dr. Manuel María Giménez" de la ciudad de Resistencia (Argentina) y que voluntariamente accedieron a la realización del estudio para diagnóstico y tipificación de HPV. Luego del consentimiento informado de los pacientes, se confeccionó una ficha epidemiológica, se realizó un examen clínico general y se tomaron muestras genitales y orales. Se excluyeron aquellos pacientes que asistieron para control del tratamiento viral o que no accedieron a la realización del estudio.

Recolección y conservación de las muestras: Se procedió a efectuar la toma de muestras por raspado con cepillos citológicos de las lesiones verrugosas anogenitales y orales; cuando no se observaron lesiones a este nivel se realizó un hisopado de la cavidad oral (mucosa yugal, piso de boca, dorso de lengua, paladar duro). Las células obtenidas por cepillado o hisopado fueron recogidas en buffer fosfato salino (PBS) estéril de $\mathrm{pH} 7,4$ y conservadas a $4{ }^{\circ} \mathrm{C}$ hasta su envío al laboratorio, dentro de las 24 horas de recogidas.

Extracción de ácidos nucleicos: Las células fueron centrifugadas y lavadas con PBS. EI ADN fue extraído según un método previamente descripto ${ }^{(11)}$. El material genético obtenido fue conservado a $-20^{\circ} \mathrm{C}$ hasta su procesamiento.

Control de calidad del ADN: La integridad del ADN obtenido fue verificada mediante la amplificación por PCR de un fragmento de 268 pares de bases (pb) del gen de la $\beta$ globina humana, empleando los cebadores $\mathrm{GH} 20$ y $\mathrm{PCO} 4^{(12)}$

Detección y tipificación de HPV: se realizó la amplificación por PCR genérica usando los cebadores degenerados MY9 (5'-CGTCCMARRGGAWACTGATC-3') y MY11 (5'GCMCAGGGWCATAAYAATGG-3') que amplifican un fragmento de 450 pb del gen L19(12); la tipificación se efectuó por análisis de los patrones de bandas obtenidos mediante la restricción enzimática de los fragmentos amplificados (RFLP) empleando las endonucleasas BamHI, Ddel, HaellI, Hinfl, Pstl, Rsal y Sau3Al en forma separada ${ }^{(12)}$. Cuando la PCR inicial resultó negativa o mostró productos inespecíficos que impedían su posterior tipificación por RFLP, las muestras fueron amplificadas usando los cebadores genéricos Gp5+ (5'-TTTGTTACTGTGGTAGATACTAC-3') y Gp6+ (5'-GAAAAATAAACTGTAAATCATATTC-3') que producen un fragmento de $140 \mathrm{pb}$ dentro de la misma región ${ }^{(12)}$; en este caso, la tipificación posterior se realizó por hibridación en dot blot con oligosondas de los tipos $6,11,16,18,31$ y 33, marcadas con biotina y la detección de los híbridos se llevó a cabo por colorimetría(13).

Análisis estadístico: Los datos obtenidos fueron almacenados y procesados utilizando el programa Epi Info®.

\section{RESULTADOS}

En total se estudiaron 26 pacientes masculinos que presentaron lesiones anogenitales cuyas edades estaban comprendidas entre 17 y 81 años (Media $=30$ años, Mediana $=28$ años). La edad promedio de inicio de relaciones sexuales fue de 17 años.

La prevalencia de HPV oral en pacientes con HPV anogenital fue de 12/26 (46,2\%) hallándose lesiones vegetantes en piso de boca en 7 de ellos.

En cuanto a la tipificación del HPV en cavidad oral (Tabla 1), 18 resultaron ser de alto riesgo oncogénico en infección única o con coinfección, siendo el tipo 16 el más frecuente ( 6 casos), seguido de los genotipos 18 y 33 ( 4 muestras cada uno), 31 y 45 
( 2 muestras cada uno) y de bajo riesgo resultaron 14, siendo los tipos más frecuentes el 6 (10 casos) y el 11 ( 4 casos).

Con relación a las conductas de riesgo, no todos los pacientes refirieron tener relaciones sexuales con otros hombres y entre los pacientes con HPV oral, todos afirmaron practicar sexo oral y haber tenido más de 5 parejas sexuales; 8 de los pacientes afirmaron ser tabaquistas.

El genotipo anogenital más frecuente fue el HPV 6 seguido por el 11, presentándose solos o en coinfección (Tabla 1).

Tabla 1. Genotipos de Papilomavirus Humano (HPV) según la región en la que fueron detectados

\begin{tabular}{ccc}
\hline Genotipo & Mucosa oral & Región anogenital \\
\cline { 2 - 3 } & $\mathbf{n}$ & $\mathbf{n}$ \\
\hline 6 & 13 & 10 \\
11 & 9 & 4 \\
16 & 6 & 6 \\
18 & 3 & 3 \\
31 & 3 & 2 \\
33 & 4 & 4 \\
45 & 4 & 2 \\
\hline
\end{tabular}

\section{DISCUSIÓN}

Según la bibliografía consultada, de los más de 100 genotipos del papilomavirus humano, se han encontrados 16 en lesiones bucales: $1,2,3,4,6,7,10,11,13,16$, $18,31,32,33,35$ y 57 ; el 13 y 32 son exclusivos de la cavidad bucal ${ }^{(3)}$. La mayoría de esos virus son de bajo riesgo (no oncogénicos) asociados a lesiones papilomatosas benignas: el 6 y 11 están asociados a papiloma bucal, 6 y 4 a verruga vulgar, 11 al condiloma acuminado, 13 y 32 a la hiperplasia epitelial focal, y tienen bajo potencial de progresión maligna. En contraste, los genotipos de alto riesgo (oncogénicos) como el $16,18,31,33$ y 35 , están asociados con leucoplasia y carcinoma escamocelular ${ }^{(7)}$.

En hombres es importante caracterizar las infecciones genitales por HPV debido a su asociación con el cáncer anogenital y las verrugas genitales, como así también por el papel que los varones desempeñan en la transmisión de HPV a sus parejas sexuales.

En el desarrollo del cáncer bucal relacionado con el HPV es importante conocer la prevalencia del mismo en la mucosa bucal sana; este rango de detección varía de 0 a $81 \%$ según un estudio previamente publicado ${ }^{(7)}$; lo mismo sucede con la prevalencia del HPV en cáncer bucal, que va de 30 al 80\%.La mayor parte de los investigadores concuerdan que la presencia de HPV se incrementa conforme la progresión displásica, ya que en los pacientes afectados es dos veces mayor que en pacientes con mucosa normal y 11 veces más frecuente en el tejido tumoral que en la mucosa oral normal distante del mismo paciente ${ }^{(11)}$.

El HPV no puede ser cultivado in vitro y los estudios serológicos tienen una sensibilidad limitada, por lo que resulta adecuado buscar el DNA del HPV ya que además puede predecir que mujeres y hombres corren riesgo de padecer lesiones precancerosas; es por ello que en este estudio hemos utilizado la amplificación mediante PCR-RFLP con iniciadores consenso, por su mayor sensibilidad refrendada en diversas publicaciones ${ }^{(14)}$.

Los tipos virales hallados en esta población y su distribución coinciden, en rasgos generales, con los datos informados en el principal estudio internacional que señala los tipos virales más frecuentes en las lesiones preneoplásicas severas y cánceres ${ }^{(9)}$. 
Medina y cols., al estudiar 64 pacientes con verrugas anogenitales, encontraron que el tipo 6 fue el más frecuente en 41 casos (64\%) seguido por el HPV 16 en 19 casos únicos o asociados a otros tipos virales ${ }^{(15)}$.

Las infecciones múltiples son consideradas un factor de riesgo para el desarrollo de lesiones preneoplásicas ${ }^{(9)}$. En este estudio se detectó un $19,2 \%$ de coinfecciones de virus de alto y bajo riesgo. El HPV 6 y el HPV 16 fueron los tipos más frecuentes hallados, aún en coinfecciones, lo cual coincide con otros estudios ${ }^{(16)}$.

En un estudio realizado en Brasil se encontró, sobre 366 hombres estudiados, que los tipos de HPV más frecuentes fueron $6,16,42$ y 51 y la coinfección se encontró en el $59 \%$ de los pacientes ${ }^{(17)}$.

Otros autores encontraron que, en Colombia, HPV 6 fue el tipo más común tanto en mujeres $(62 \%)$ como en hombres $(56 \%)$, seguido de HPV $11(20 \%)^{(18)}$. La coinfección con estos dos tipos ocurrió en $7 \%$ y $12 \%$ de mujeres y hombres, respectivamente, HPV 16 ocupó el tercer lugar en prevalencia, con el 16\% de los pacientes que prueban positivos y el $25 \%$ de los casos fueron positivos para múltiples genotipos de VPH.

Cavazza Porro y cols encontraron predominancia de HPV 6 en el 58\% de los pacientes varones con verrugas genitales y de HPV 11 en el $40 \%$ de ellos ${ }^{(19)}$.

En cuanto al cáncer oral, el HPV 16 es el tipo más frecuentemente asociado, especialmente en las localizaciones de orofaringe y amígdala. Las lesiones asociadas a la infección por HPV en la cavidad oral son principalmente la papilomatosis oral (asociada a los HPV 6 y 11) y eritroplaquia (HPV 16).

El cáncer oral predomina en el género masculino, siendo de dos a quince veces más frecuente en hombres que en mujeres dependiendo de la localización anatómica ${ }^{(20,21)}$.

El tercer tipo más prevalente es el HPV 6, considerado de bajo o moderado riesgo oncogénico en base a los resultados de las investigaciones realizadas sobre carcinomas de células escamosas de cuello uterino ${ }^{(22)}$.

En los pacientes estudiados, las coinfecciones podrían estar asociadas con conductas sexuales como la práctica de sexo oral, el elevado número de parejas y el inicio temprano de las relaciones sexuales, lo que podría aumentar el riesgo de persistencia viral y progresión maligna de las lesiones.

Otro de los factores fuertemente asociados al cáncer oral es el consumo de tabaco y de bebidas alcohólicas ${ }^{(23)}$ y estas afirmaciones coinciden con nuestros hallazgos.

Aunque en el presente estudio se encontró una edad media de 30 años de edad para los pacientes infectados con HPV, la mayoría de estos pacientes fueron menores de 30 años, lo cual coincide con lo demostrado por diversos autores ${ }^{(24)}$.

El inicio precoz de las relaciones sexuales también es considerado un factor de riesgo para la infección por HPV y en este estudio se halló que la edad media de inicio de relaciones sexuales fue de 17 años, con un mínimo de 13 años; esto coincide con los datos aportados por Bianco y col, quienes encontraron que en la Argentina la mayoría de los varones encuestados se inició sexualmente a los 14,4 años y la mayoría de las mujeres a los 15,2 años ${ }^{(25)}$.

Por otro lado, se observó que todos los pacientes estudiados tuvieron más de 5 contactos sexuales, lo cual es otro factor de riesgo para la adquisición de la infección persistente por tipos de alto riesgo de HPV y el mayor riesgo de progresión a distintos grados de lesión ${ }^{(24)}$.

La infección por HPV entre los hombres que tienen relaciones sexuales con hombres (HSH) es el principal factor de riesgo para el desarrollo del cáncer anal, una condición que supera a la prevalencia del cáncer cervical en las mujeres ${ }^{(26)}$.

En el presente trabajo solamente el $10 \%$ de los pacientes refirió tener sexo con hombres.

En el estudio realizado por Quin y col, sobre $105 \mathrm{HSH}$ peruanos estudiados, el $77,1 \%$ se infectó con HPV, de éstos el $79 \%$ se coinfectó con dos o más tipos y el $47,3 \%$ se infectó con un tipo carcinogénico ${ }^{(27)}$. Los tipos de HPV 53, 6, 16 y 58 fueron los más frecuentemente detectados.

Como se ha mencionado, la infección por HPV es necesaria pero no suficiente para que se produzca la transformación oncogénica ${ }^{(16)}$, sino que se necesita la asociación de diferentes factores a los antes mencionados para el desarrollo del cáncer oral ${ }^{(22)}$. 
EI DNA del virus del papiloma humano ha sido demostrado en algunos carcinomas de células escamosas sin embargo, hay poca información de la prevalencia en la mucosa bucal clínicamente sana ${ }^{(24)}$.

Respecto de las formas clínicas encontradas en cavidad oral, las más frecuentes fueron las vegetantes $(15,4 \%)$ y localizadas en piso de boca $(15 \%)$, lo cual es concordante con lo informado por otros autores ${ }^{(22,23,24)}$.

Los datos sobre la distribución de tipos específicos de HPV pueden ser útiles para evaluar los beneficios adicionales de las vacunas, la protección cruzada contra los tipos no vacunales, la inmunidad de rebaño y para monitorear los fenómenos adversos $^{(28)}$

En un estudio realizado en Londres sobre muestras anogenitales de $\mathrm{HSH}$, se encontró que la prevalencia de los tipos de HPV prevenibles mediante la vacuna fue del $17,0 \%, 32,5 \%$ y $45,4 \%$ para la bivalente, cuadrivalente y nonavalente, respectivamente. Esto indica que debería estudiarse la inclusión de otros tipos virales para la población con esa conducta de riesgo.

Los tipos virales hallados por nosotros coinciden con los prevalentes a nivel mundial por lo que la vacuna polivalente que está basada en estos genotipos virales sería efectiva en la población incluida en el presente trabajo ${ }^{(29,30)}$.

Nuestros resultados proporcionan evidencias acerca de la alta prevalencia de infección por HPV en hombres lo cual podría explicar la alta frecuencia de cáncer oral y cervical en las mujeres y de cáncer de pene en los hombres en nuestra región.

\section{CONCLUSIÓN}

Se halló una alta prevalencia de infección por HPV y una elevada proporción de coinfección con distintos tipos oncogénicos en la población menor de 30 años.

Los aquí presentados son los primeros datos sobre los tipos virales predominantes en muestras orales y anogenitales en la Región del Nordeste de la República Argentina en población masculina.

Este estudio se considera un aporte a la epidemiología de la infección por HPVHPV en una región de Argentina, información que resulta imprescindible para el diseño de Programas de Prevención del Cáncer Cervical, Anogenital y Orofaríngeo.

\section{Conflicto de interés}

Los autores declaran la no existencia de conflicto de intereses.

\section{Financiamiento}

El presente estudio se llevó a cabo con fondos genuinos de las instituciones a la que pertenecen los autores.

\section{Contribución de los autores}

Alicia Sorrentino: diseño del trabajo y procesamiento de muestras; Luis A. Merino: diseño del trabajo, análisis de resultados y redacción del manuscrito; Marcelo G. Medina: recolección de muestras, análisis de resultados y redacción del manuscrito; Myriam L. Medina: análisis de resultados y revisión final del manuscrito; Karina Marinic: diseño del trabajo y procesamiento de muestras; Manuel F. Giménez: diseño del trabajo y análisis de resultados.

\section{REFERENCIAS BI BLI OGRÁFI CAS}

1. De Villiers EM, Fauquet C, Broker TR, Bernard HU, Zur Hausen $H$. Classification of papillomaviruses. Virology. 2004; 324(1): 17- 27.

2. Parkin DM. The global health burden of infection-associated cancers in the year 2002. Int J Cancer. 2006; 118(12): 3030- 44.

3. Muñoz N, Bosch FX, de Sanjosé S, Herrero R, Castellsagué X, Shah K V., et al. Epidemiologic Classification of
Human Papillomavirus Types Associated with Cervical Cancer. N Engl J Med. 2003; 348(6): 518- 27

4. Walczak I, Dutkiewicz S, Majewski S, Jablonska S, Witeska A. Diagnosis of Human Papillomavirus (HPV): infection of male genital skin and mucosa. Urology. 2008; 72(5): S34.

5. Van Oijen MGCT, Leppers Vd Straat FGJ, Tilanus MGJ, Slootweg PJ. The origins of multiple squamous cell 
carcinomas in the aerodigestive tract. Cancer. 2000; 88(4): 884-93.

6. Zeuss MS, Miller CS, White DK. In situ hybridization analysis of human papillomavirus DNA in oral mucosal lesions. Oral Surgery, Oral Med Oral Pathol. 1991; 71(6):714-20.

7. Sugiyama M, Bhawal UK, Dohmen T, Ono S, Miyauchi $M$, Ishikawa $T$. Detection of human papillomavirus-16 and HPV-18 DNA in normal, dysplastic, and malignant oral epithelium. Oral Surg Oral Med Oral Pathol Oral Radiol Endod. 2003; 95(5): 594-600.

8. de Villiers EM. Heterogeneity of the human papillomavirus group. J Virol. 1989; 63(11): 4898-903.

9. Frazer $\mathrm{IH}$, Cox JT, Mayeaux EJ, Franco EL, Moscicki AB, Palefsky JM, et al. Advances in prevention of cervical cancer and other human papillomavirus-related diseases. Pediatr Infect Dis J. 2006; 25(2 SUPPL.): 6581.

10. Silva R, León D, Brebi P, Ili C, Roa JC, Sánchez R. Detection of human papilloma virus infection in men. Rev Chil infectología. 2013; 30(2): 186-92.

11. Bernard HU, Chan SY, Manos MM, Ong CK, Villa LL, Delius $H$, et al. Identification and assessment of known and novel human papillomaviruses by polymerase chain reaction amplification, restriction fragment length polymorphisms, nucleotide sequence, and phylogenetic algorithms. J Infect Dis. 1994; 170(5): 1077-85.

12. Wheeler C, Yamada T. Human papillomavirus type 16 sequence variants: identification by $\mathrm{E} 6$ and $\mathrm{L} 1$ Human Papillomavirus Type 16 Sequence Variants: Identification by E6 and L1. J Clin Microbiol. 1997; 35(1): 11-9.

13. Molijn A, Kleter B, Quint W, Van Doorn LJ. Molecular diagnosis of human papillomavirus (HPV) infections. J Clin Virol. 2005; 32(SUPPL.):43-51.

14. Mandelblatt JS, Lawrence WF, Womack SM, Jacobson D, Yi B, Hwang YT, et al. Benefits and costs of using HPV testing to screen for cervical cancer. J Am Med Assoc. 2002; 287(18): 2372-81.

15. Medina MG, Marinic K, Motta $P$, Sorrentino A, Giménez MF. Detección y genotipificación de papilomavirus humano en hombres. Piel. 2010;25(10): 561-4.

16. Partridge JM, Koutsky LA. Genital human papillomavirus infection in men. Lancet Infect Dis. 2006; 6(1):21-31.

17. Freire MP, Pires D, Forjaz R, Sato S, Cotrim I, Stiepcich M, et al. Genital prevalence of HPV types and co- infection in men. Int Braz J Urol. 2014; 40(1):67-71.

18. Hernandez-Suarez G, Pineros M, Vargas J C, Orjuela L, Hernandez F, Peroza C, et al. Human papillomavirus genotypes in genital warts in Latin America: A crosssectional study in Bogota, Colombia. Int J STD AIDS. 2013; 24(7):567-72.

19. Cavazza Porro M, Avila M, Correnti $M$, Vasquez $W$, López $Y$. Human Papillomavirus in Venezuelan patients with condylomata acuminata. In: 24th International Papillomavirus Conference and Clinical Workshop [Internet]. Beijin: International Papillomavirus Society; 2007. p. PS12-04. Available from: http://af.global.cnki.net/stmt/Titl eBrowse/KnowledgeNet/ZHYX20071100 $5462 ? \mathrm{db}=$ STMI 8320

20. Stewart B, Kleihues $P$, editors. World Cancer Report. WHO Chronicle. Lyon: IARC Press; 2003. 323-326 p.

21. Lipsky MS, Su S, Crespo CJ, Hung M. Men and Oral Health: A Review of Sex and Gender Differences. Am J Mens Health. 2021; 15 (3).

22. Kreimer AR, Clifford GM, Boyle P, Franceschi S. Human papillomavirus types in head and neck squamous cell carcinomas worldwide: A systemic review. Cancer Epidemiol Biomarkers Prev. 2005; 14(2): 467-75.

23. Herrero $R$, Castellsagué $X$, Pawlita $M$, Lissowska J, Kee $F$, Balaram $P$, et al. Human papillomavirus and oral cancer: The international agency for research on cancer multicenter study. J Natl Cancer Inst. 2003; 95(23): 1772-83.

24. Schiffman $M H$, Bauer HM, Hoover RN, Glass AG, Cadell DM, Rush BB, et al. Epidemiologic evidence showing that Human Papillomavirus infection causes most cervical intraepithelial neoplasia. J Natl Cancer Inst. 1993 Jun 16; 85(12): 958-64.

25. Bianco M, Correa C. La adolescencia en Argentina: sexualidad y pobreza [Internet]. CABA; 2003. Available from: http: //feim.org. ar/2003/06/14/laadolescencia-en-argentina-sexualidady-pobreza/

26. Nyitray AG, Carvalho Da Silva RJ, Baggio ML, Lu B, Smith D, Abrahamsen $M$, et al. Age-specific prevalence of and risk factors for anal human papillomavirus (HPV) among men who have sex with women and men who have sex with men: The HPV in men (HIM) study. J Infect Dis. 2011; 203(1): 49- 57.

27. Quinn R, Salvatierra J, Solari V, Calderon M, Ton TGN, Zunt JR. Human papillomavirus infection in men who have sex with men in Lima, Peru. AIDS 
Res Hum Retroviruses. 2012; 28(12): 1734-8.

28. González JV, Deluca GD, Liotta DJ, Correa RM, Basiletti JA, Colucci MC, et al. Baseline prevalence and type distribution of Human papillomavirus in sexually active non-vaccinated adolescent girls from Argentina. Rev Argent Microbiol. 2021; 53 (1): 11-9.
29. Aranda CE. Infección por el virus del papiloma humano en varones. Ginecol Obstet Mex. 2015; 83(11):697-706.

30. Morelatto RA, Lopez De Blanc SA. Oral cancer mortality in the province of Cordoba, Argentine Republic (period 1975-2000). A comparative study with other populations. Med Oral Patol Oral Cir Bucal. 2006; 11(3):144-9. 